\title{
Real-time Traffic Monitoring by fusing Floating Car Data with Stationary Detector Data
}

\author{
Maarten Houbraken, \\ Pieter Audenaert, Didier Colle and Mario Pickavet \\ Department of Information Technology \\ Ghent University \\ Ghent, Belgium \\ E-mail: maarten.houbraken@intec.ugent.be
}

\author{
Karolien Scheerlinck, \\ Isaak Yperman and Steven Logghe \\ Be-Mobile \\ Melle, Belgium
}

\begin{abstract}
Applying the current technological possibilities has led to a wide range of traffic monitoring systems. These heterogeneous data sources individually provide a view on the current traffic state, each source having its own properties and (dis)advantages. However, these different sources can be aggregated to create a single traffic state estimation. This paper presents a data fusion algorithm that combines data on the data sample level. The proposed system fuses floating car data with stationary detector data and was implemented on live traffic. Results show the fusion algorithm allows to eliminate individual source bias and alleviates source-specific limitations.
\end{abstract}

Keywords-floating car data; stationary detector data; data fusion; traffic state estimation

\section{INTRODUCTION}

With the advances in communication technology and location-aware devices, more and more cars connect to or use Intelligent Transportation Systems (ITS) to get real-time traffic information and optimize their journey. These real-time ITS and their underlying traffic models vary depending on the available inputs, specific use-case and intended features. However, all these models need accurate and real-time traffic state information to assess the current traffic conditions. In this work, we focus on large scale traffic state estimation using both realtime floating car data and stationary detector data.

Floating car data (FCD) denotes data generated by moving vehicles called probes. These vehicles collect data with on-board positioning devices that record their position (often at a fixed interval). This data is then transmitted to a data collection server that processes the data for all probes (see $[1,2]$ for details on sample processing). From there on, it can be used for a wide range of applications. One of the earliest consisted of calculating average travel times on routes throughout the day [3-5]. Note that this does not necessarily require real time sample transmission/processing as it can be done using historic data. However, the main strength of FCD lies in its real time applications. Real time sample aggregation and processing can be used for live traffic state detection [2] and estimation [6].
Stationary detector data (SDD) consists of data captured on fixed points in the road network by cameras, embedded inductive loops or other measuring equipment. This offers high resolution monitoring of the specific location (often with samples generated every 10-60 seconds). A full view on the traffic state along the trajectory can be made by combining the SDD from several points with macroscopic flow theory. Kalman filtering models the traffic as flows of cars and estimates the traffic state space for each time period [7]. Further extensions to the model have been made to account for irregular sample generation by using multiple modes of filter operation [8].

While both sources separately can create a full traffic view, their combination allows a more detailed and accurate estimation of the traffic state. This is called data fusion and aims to fully capture the information in the individual sources. While SDD provides a detailed view on the traffic state on the monitored locations, installing and maintaining these sensors on the entire road network would require high investments. FCD is more easily used for this type of traffic monitoring as it distributed design inherently monitors large geographical areas instead of fixed locations. However, FCD accuracy depends on the number of monitored probe vehicles, often limited to a small fraction of the total traffic.

Data fusion itself has be done with a wide range of techniques, e.g. neural networks [9-12] or Kalman modelling [13], fusing additional data sources e.g. automatic license plate recognition [14]. More example techniques and an overview of applications can be found in $[15,16]$. In this paper, we focus on an adaptive smoothing technique proposed in $[17,18]$, also known as the extended and generalized Treiber-Helbing filter (EGTF). This filter uses kinematic wave theory applied to road traffic to merge individual samples to a full traffic state estimation.

\section{BASIC DATA FUSION ALGORITHM}

For this EGTF technique, the road network is modelled as a dynamic system in which traffic flows along the roads, with cars driving from position $A$ at time $t_{A}$ to position $B$ at time $t_{B}$. In freeflow traffic, the traffic conditions move along with the traffic, meaning that the traffic condition at position $A$ at time $t_{A}$ will be 
2015 Models and Technologies for Intelligent Transportation Systems (MT-ITS)

very similar to the traffic conditions at position $B$ at time $t_{B}$. This implies that the traffic state at position $B$ at a certain time $t_{x}$ (possibly in the near future) can be predicted by using a traffic state from position $A$ at an earlier time $t_{y}$.

In congested traffic, however, the traffic conditions move in the opposite direction of the traffic. While the traffic at the head of a traffic jam starts to move again, the back of the traffic jam is still stationary, with even more cars queuing on. This results in the head of the jam moving backwards and causes stop-andgo waves. More importantly, the prediction of the traffic state at position $A$ should take into account the traffic state at position $B$ (further along the highway in the normal driving direction).

These two mechanisms are modelled as separate waves in the Treiber-Helbing filter, moving at different speeds and in different directions. A schematic representation of the system is shown in Fig. 1. To estimate the traffic state for the position denoted by the diamond, the loop samples (denoted by triangles) on positions further along the route are taken into account by the backward propagating wave (denoted by the ellipsoid going from the top left to the bottom right). Analogously, the loop samples contributing to the forward wave are found in the forward ellipsoid (bottom left to top right). Following the above reasoning, the data samples within both ellipses are considered most relevant to the traffic state and will therefore be weighted more in the estimation of the traffic state at position $P$.

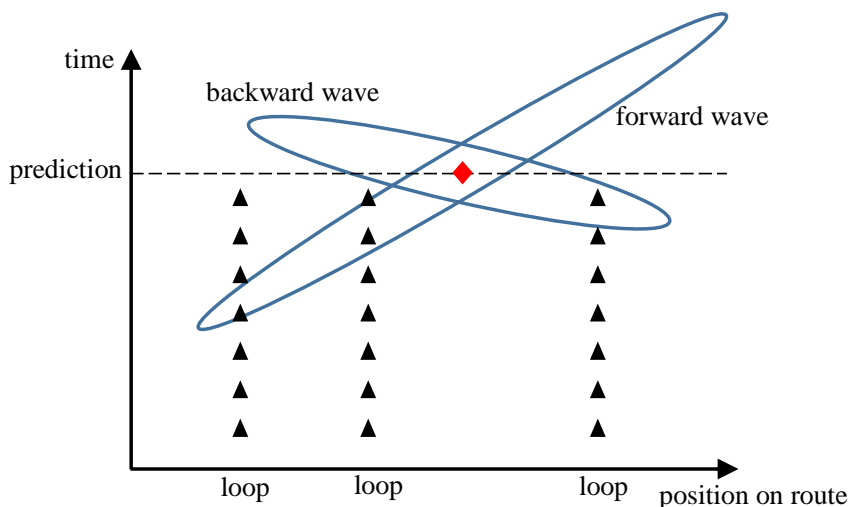

Figure 1: Schematic representation of the traffic wave in the EGTF

To calculate the traffic state, the available samples are processed in a weighted sum. The estimated speed $s(x, t)$ at position $x$ and time $t$ for one wave is then calculated as a weighted sum of the speed of all samples in the region of interest around the $(x, t)$ point. This weighted sum is given by

$$
s(x, t)=\sum_{i}\left(w_{i}(x, t) \cdot s_{i}\right) / \sum_{i} w_{i}(x, t)
$$

with $w_{i}(x, t)$ the weight of a sample at position $x_{i}$, taken at time $t_{i}$, contributing to the state at position $x$ at time $t$. The individual weights $w_{i}(x, t)$ are calculated as follows

$$
w_{i}(x, t)=\exp \left(-\left|x-x_{i}\right| / \sigma-\left|t-t_{i}-\left(\left(x-x_{i}\right) / v\right)\right| / \tau\right)
$$

with $\sigma$ and $\tau$ denoting the width and time window, respectively, of the region of influence around the $(x, t)$ point under estimation. This weight function favors samples according to the ellipsoids shown in Fig. 1. The $\sigma$ and $\tau$ are tuned to the availability of samples, which depends on the average distance between installed detectors and their sampling interval. The $v$ in the formula denotes the propagation speed of the wave and differs between the forward free-flow wave $\left(v_{F F}\right)$ and the backward wave $\left(v_{C O N G}\right)$. This results in 2 speed estimates $s_{F F}(x, t)$ and $s_{C O N G}(x, t)$.

To obtain a single speed estimate $v(x, t)$ from the 2 estimates, they are combined using the following equations

$$
\begin{gathered}
v(x, t)=z(x, t) \cdot s_{C O N G}(x, t)+(1-z(x, t)) \cdot s_{F F}(x, t) \\
z(x, t)=1 / 2 \cdot\left(1+\tanh \left(\left(V_{c}-\min \left(s_{C O N G}(x, t), s_{F F}(x, t)\right)\right) / d V\right)\right.
\end{gathered}
$$

with $V_{c}$ the speed at which free-flow traffic transitions to congested traffic and $d V$ the sensitivity around this threshold.

In Eq. 4, the two speed values (from the different propagating waves) are combined to estimate whether the traffic is in congestion $(z>1 / 2)$ or in free-flow $(z<1 / 2)$. If $s_{\text {cong }}(x, t)$ or $s_{F F}(x, t)$ are below the transition threshold, the congested estimate becomes more dominant in the calculation of the final speed estimate $v(x, t)$.

\section{DATA SOURCE NORMALISATION}

The above calculation merges individual samples to a full traffic state estimate. However, when fusing different data sources like FCD and SDD, the individual samples from both sources have different fundamental properties, as they are generated using different measuring techniques. In the EGTF, this difference is taken into account by first calculating speed estimates for each source and then combining them using an extra weight factor denoting the estimated confidence/accuracy of the speed estimate. However, the fusion process can be done on the sample level, thereby needing only a single THF. The only requirement is that the individual samples from the different sources are taken into account equally to avoid bias towards an individual source. In the case of FCD and SDD, a correction is needed as both generate samples with different properties and frequency.

SDD typically consists of samples taken with a fixed frequency (e.g. 1 sample per minute). However, this fixed interval can vary from detector to detector. This would result in some detectors producing more samples in the same time interval and therefore being favored in the sample aggregation. Analogously, this frequency also needs to be tuned to the FCD generation which uses full trips (or trips in progress) to generate data samples, possibly multiple samples if the trip passes multiple road segments. This can be solved for each source individually by tuning the frequency of the sample generation and their importance in the sample weighting. By equalizing the number of samples and their contribution to the calculated estimates, the estimation avoids favoring a specific source. 
2015 Models and Technologies for Intelligent Transportation Systems (MT-ITS)

3-5. June 2015. Budapest, Hungary

Depending on the source (and individual detector), the specific normalization also needs to take into account the specific source measuring properties. SDD consists of data measured at fixed positions in the network. In contrast, FCD originates from tracking vehicles. The reported values in SDD are therefore inherently temporal averages while the FCD reports spatial averages (see [19] for a more detailed description of the difference). To merge the reported values, they need to be converted to the same averaging (spatial/temporal depending on the desired estimation) before being summed.

A final normalization is needed to account for the coverage provided in the data sources. While SDD provides aggregated data for all vehicles, FCD only covers a small fraction of the total traffic on the road. This results in different absolute numbers of samples, varying according with traffic intensity as well as the FCD probe vehicle penetration rate. This can be solved by taking the penetration rate into account in the weighting.

\section{EXPERIMENTAL APPLICATION: A58 USE CASE}

To validate the above method, the system was implemented for the $20 \mathrm{~km}$ of A58 highway from Tilburg to Eindhoven shown in Fig. 2. While the entire area of study in the 'Spookfiles' project (www.spookfiles.nl) entailed the neighboring A2 and A67, only results for the A58 are given here.

The A58 is monitored by 33 dual induction loops spaced evenly over the highway, resulting in available SDD roughly every $500 \mathrm{~m}$. The live data from these loops was collected every minute and processed real-time to obtain the results below. The FCD was supplied by Be-Mobile, obtained from monitoring an estimated $3 \%$ of all traffic on the highway, higher than the $1.5 \%$ reported in [2]. Note that as the data is collected real-time, there needs to be accounted for delay, caused by the sample averaging of the measurement equipment, communication overhead and processing time.

Fig. 3 shows the output of the SDD plotted at their respective positions along the route. This data was combined with the aggregated FCD (depicted in Fig. 4) and processed using the THF described above. The results are plotted in Fig. 5. At 6:00 AM, the traffic on the route is smooth. The first congestion starts to form around 6:50 AM near the Moergestel exit. This congestion propagates backwards (as a stop-and-go wave) to the start of the route (where 2 highways merge to form the A58) and reinforces the jam that was forming there. Around 7:00 AM, a congestion wave also start at the end of the route. This wave also propagates backwards and reaches the Oirschot intersection around 7:30 AM. This causes the traffic there to become disturbed for the rest of the morning rush hour. On top of the first wave, 2 more congestion waves propagate backward to Moergestel.

Comparing the SDD source to the fusion result, the fusion algorithm more clearly captures the traffic state when the distance between individual detectors becomes larger (e.g. between the start of the route and the Tilburg entry) or when traffic is turbulent (e.g. between the Moergestel exit and the preceding rest stop). Compared to the FCD source, the fusion algorithm provides a smoother traffic state with a clearer distinction between individual waves (e.g. between Tilburg and Moergestel).

The SDD provides accurate speed observations but is limited in spatial resolution compared to the FCD. The FCD in turn fluctuates very rapidly (as shown by the sharp color differences in Fig. 4). The data fusion processing combines both data sources in a smooth traffic state. Note again that the prediction for a specific time and position is made using only older samples as data is collected and processed real-time.

\section{CONCLUSION AND FUTURE WORK}

With more and more heterogeneous traffic monitoring, the traffic state estimation has to incorporate these individual sources in a consistent framework. By normalizing the individual data samples to take into account the measuring properties, they can be combined in a single data fusion algorithm to estimate traffic state. This approach was applied to fuse stationary detector data with floating car data, both gathered and processed real-time. Results show that this corrects individual data source bias, resulting in a cleaner traffic state estimation.

While our study was limited to the well-monitored A58 highway, most road infrastructure is not equipped with traffic sensors, thereby limiting the availability of SDD. However, the data fusion algorithm does not require a single source to provide a full traffic view. As samples from different sources can be merged, the algorithm can be applied to roads with fewer sensors. More interestingly, it may also be used to avoid having to install an extensive network of detectors, thereby lowering infrastructure cost.

\section{ACKNOWLEDGMENT}

Maarten Houbraken is supported by a $\mathrm{PhD}$ fellowship grant by the Research Foundation Flanders (FWO-Vlaanderen). The authors would also like to thank Be-Mobile for their cooperation and supplying the traffic data.

\section{REFERENCES}

[1] M. Vanlommel, M. Houbraken, P. Audenaert, S. Logghe, M. Pickavet, and P. De Maeyer, "An evaluation of section control based on floating car data", Transportation Research Part C: Emerging Technologies, in press, 2015

[2] B. S. Kerner, C. Demir, R. G. Herrtwich, S. L. Klenov, H. Rehborn, M. Aleksic, and A. Haug, "Traffic state detection with floating car data in road networks", Proc. 2005 IEEE Intelligent Transportation Systems, pp. 44-49, September 2005.

[3] M. Vanlommel, P. De Maeyer, and S. Logghe, "Dynamical network management and historical analysis based on floating car data", $2^{\text {nd }}$ international conference on Models and Technologies in Intelligent Transportation Systems, june 2011.

[4] E. Brockfeld, B. Passfeld, and P. Wagner, "Validating travel times calculated on the basis of taxi floating car data with test drives", Proc. 14th World Congress on Intelligent Transport Systems, October 2007.

[5] M. Reinthaler, B. Nowotny, F. Weichenmeier, and R. Hildebrandt, "Evaluation of speed estimation by floating car data within the research project DMotion", 14th World Congress on Intelligent Transport Systems, 2007. 
2015 Models and Technologies for Intelligent Transportation Systems (MT-ITS)

3-5. June 2015. Budapest, Hungary

[6] C. De Fabritiis, R. Ragona, and G. Valenti, "Traffic estimation and prediction based on real time floating car data", 11th International IEEE Conference on Intelligent Transportation Systems, October 2008, pp. 197-203.

[7] Y., Wang, and M. Papageorgiou, "Real-time freeway traffic state estimation based on extended Kalman filter: a general approach", Transportation Research Part B: Methodological, vol. 39(2), pp. 141-167. 2005.

[8] T. Tettamanti, M. T. Horváth, and I. Varga, "Road Traffic Measurement and Related Data Fusion Methodology for Traffic Estimation", Transport and Telecommunication Journal, vol. 15(4), pp. 269-279, 2014.

[9] J. N. Ivan, "Neural network representations for arterial street incident detection data fusion", Transportation Research Part C: Emerging Technologies, vol. 5(3), pp. 245-254, 1997.

[10] H. Dia, and K. Thomas, "Development and evaluation of arterial incident detection models using fusion of simulated probe vehicle and loop detector data", Information Fusion, vol. 12(1), pp. 20-27, 2011.

[11] Q. Zhang, and B. Li, "A low-cost GPS/INS integration based on UKF and BP neural network", $5^{\text {th }}$ International IEEE Conference on Intelligent Control and Information Processing (ICICIP), pp. 100-107, August 2014.

[12] Q. Ou, H. van Lint, and S. P. Hoogendoorn, "Fusing heterogeneous and unreliable data from traffic sensors", Interactive Collaborative Information Systems, pp. 511-545, 2010.
[13] E. Cipriani, S. Gori, L. Mannini, and S. Brinchi, "A procedure for urban route travel time forecast based on advanced traffic data: case study of Rome", 17th International IEEE Conference on Intelligent Transportation Systems (ITSC), pp. 936-941, October 2014.

[14] M. Rahmani, E. Jenelius, and H. N. Koutsopoulos, "Floating car and camera data fusion for non-parametric route travel time estimation", 17th International IEEE Conference on Intelligent Transportation Systems (ITSC), pp. 1286-1291, October 2014.

[15] D. Schmidt, B. Friedrich, and F. Weichenmeier, "Data fusion techniques for traffic state estimation-DINO within DMotion", 15th World Congress on Intelligent Transport Systems, pp. 15-20, November 2008.

[16] N. E. El Faouzi, H. Leung, and A. Kurian, "Data fusion in intelligent transportation systems: Progress and challenges-A survey", Information Fusion, vol. 12(1), pp. 4-10, 2011.

[17] M. Treiber, and D. Helbing, "Reconstructing the spatio-temporal traffic dynamics from stationary detector data”,Cooper@ tiveTr@ nsport@ tion Dyn@ mics, vol. 1(3), 2002.

[18] J. W. C. Van Lint, and S. P. Hoogendoorn, "A robust and efficient method for fusing heterogeneous data from traffic sensors on freeways", Computer-Aided Civil and Infrastructure Engineering, vol. 25(8), pp. 596-612, 2010

[19] M., Treiber, and A. Kesting, "Traffic Flow Dynamics: Data, Models and Simulation”, Springer Science \& Business Media. 2012.

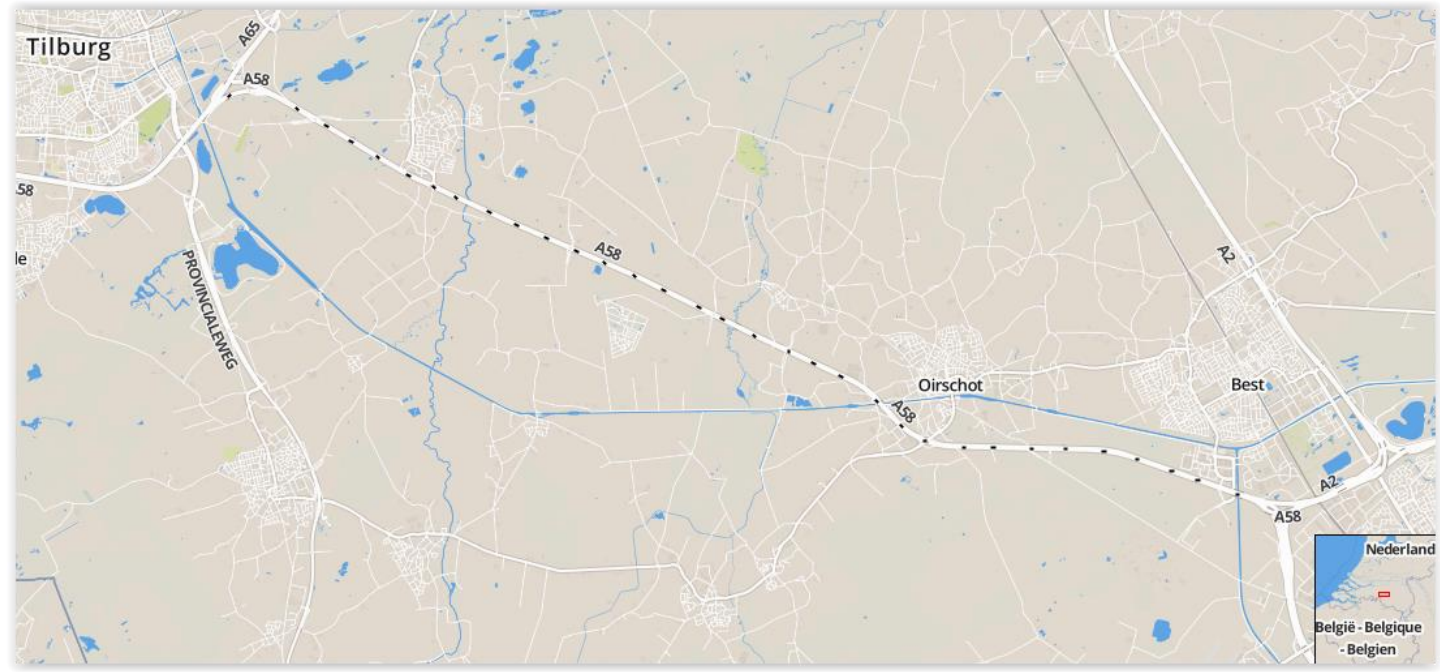

Figure 2: A58 highway. The highway spans $20 \mathrm{~km}$ and contains 33 loop detectors, indicated in black

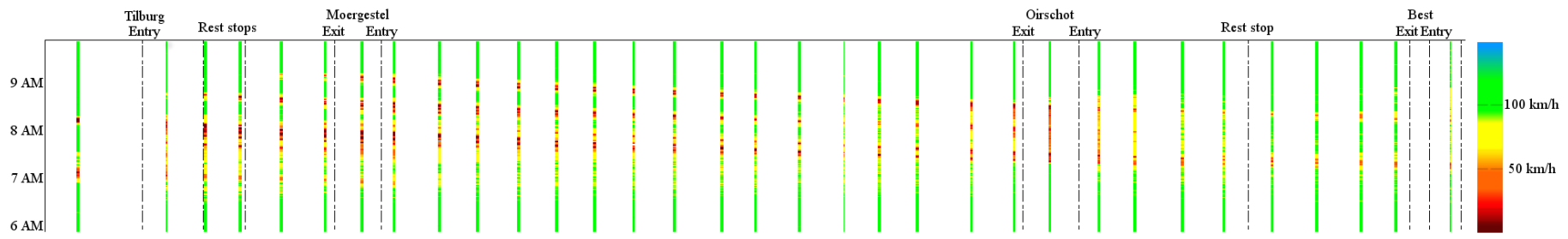

Figure 3: SDD on the A58 on $9^{\text {th }}$ February 2015 between 6 and 10 AM (local time)

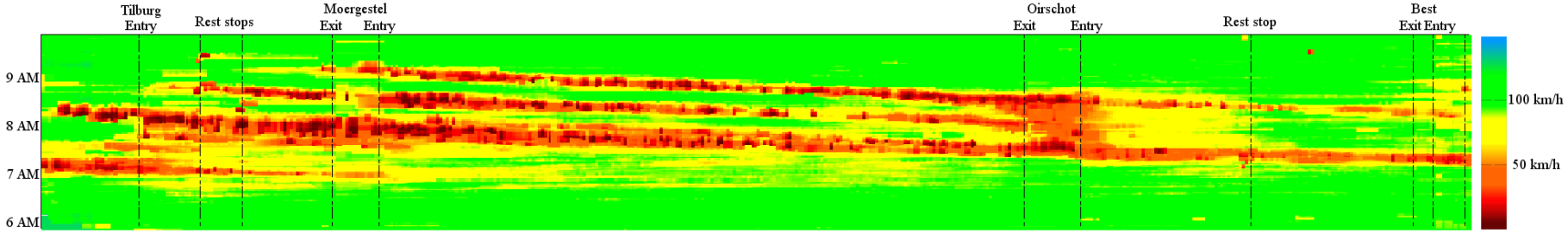


2015 Models and Technologies for Intelligent Transportation Systems (MT-ITS)

3-5. June 2015. Budapest, Hungary

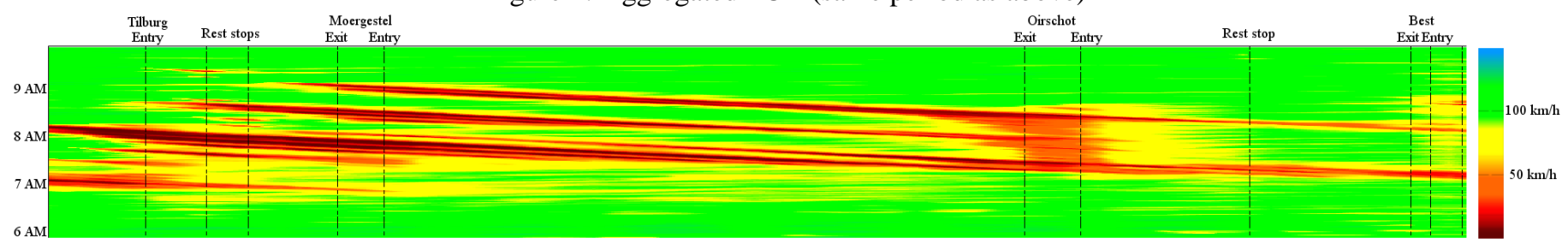

Figure 5: Fused traffic state (same period as above) 